THEORY OF COMPUTING

\title{
On the Existence Problem of Finite Bases of Identities in the Algebras of Recursive Functions
}

$\frac{\text { V. A. Sokolov }{ }^{1}}{{ }^{1} \text { P. G. Demidov Yaroslavl State University, } 14 \text { Sovetskaya, Yaroslavl 150003, Russia. }}$

MSC2020: 03D20

Research article

Full text in Russian
DOI: $10.18255 / 1818-1015-2020-3-304-315$

Received August 21, 2020

After revision September 7, 2020

Accepted September 9, 2020

Raphael Robinson showed that all primitive recursive functions depending on one argument, and only they could be obtained from two functions $s(x)=x+1$ and $q(x)=x-[\sqrt{x}]^{2}$ by using operations of addition + , superposition * and iteration $i$. Julia Robinson proved that from the same two functions, using the addition + , superposition * and operation ${ }^{-1}$ of function inversion, one could obtain all general recursive functions (under a certain condition on the inversion operation) and all partially recursive functions. On the basis of these results, A. I. Maltsev brought into consideration the Raphael Robinson algebra of all unary primitive recursive functions and two Julia Robinson algebras: the partial algebra of all unary general recursive functions and the algebra of all unary partially recursive functions and proposed to study the properties of these algebras, including the question of the existence of finite bases of identities in these algebras. In this article we show that there is no finite basis of identities in any of the indicated algebras.

Keywords: algebras; recursive functions; identities; basis; superposition; iteration; function inversion.

\section{INFORMATION ABOUT THE AUTHORS}

Valery Anatolyevich Sokolov correspondence author orcid.org/0000-0003-1427-4937. E-mail: valery-sokolov@yandex.ru

Doctor of Science, Professor, Department of Theoretical Informatics, Centre of Integrable Systems.

Funding: The initiative program VIP-004 (state registration number AAAA-A16-116070610022-6).

For citation: V. A. Sokolov, "On the Existence Problem of Finite Bases of Identities in the Algebras of Recursive Functions", Modeling and analysis of information systems, vol. 27, no. 3, pp. 304-315, 2020. 


\section{О проблеме существования конечных базисов тождеств в алгебрах рекурсивных функций}

\section{УДК 512.57}

Научная статья

Полный текст на русском языке
Получена 21 августа 2020 г.

После доработки 7 сентября 2020 г. Принята к публикации 9 сентября 2020 г.

Рафаэль Робинсон показал, что все примитивно рекурсивные функции, зависящие от одного аргумента, и только они могут быть получены из двух функций $s(x)=x+1$ и $q(x)=x-[\sqrt{x}]^{2}$ с помощью операций сложения + , суперпозиции * и итерации $i$. Джулия Робинсон доказала, что из этих же двух функций с помощью операций сложения +, суперпозиции * и операции ${ }^{-1}$ обращения функций можно получить все общерекурсивные (при определённом условии на операцию обращения) и все частично рекурсивные функции. На основании этих результатов А. И. Мальцев ввёл в рассмотрение алгебру Рафаэля Робинсона всех одноместных примитивно рекурсивных функций и две алгебры Джулии Робинсон: частичную алгебру всех одноместных общерекурсивных функций и алгебру всех одноместных частично рекурсивных функций, и предложил исследовать свойства этих алгебр, в том числе, выяснить, существуют ли в этих алгебрах конечные базисы тождеств. В этой статье мы показываем, что конечного базиса тождеств ни в одной из указанных алгебр не существует.

Ключевые слова: алгебры; рекурсивные функции; тождества; базис; суперпозиция; итерация; обращение функции.

\section{ИНФОРМАЦИЯ ОБ АВТОРАХ}

Валерий Анатольевич Соколов автор для корреспонденции

orcid.org/0000-0003-1427-4937. E-mail: valery-sokolov@yandex.ru доктор физ.-мат. наук, профессор, кафедра теоретической информатики, Центр интегрируемых систем.

Финансирование: Инициативная НИР ВИП-004 (номер госрегистрации АААА-А16-116070610022-6).

Для цитирования: V. A. Sokolov, "On the Existence Problem of Finite Bases of Identities in the Algebras of Recursive Functions”, Modeling and analysis of information systems, vol. 27, no. 3, pp. 304-315, 2020.

(ㄷ) Соколов В. А., 2020

Эта статья открытого доступа под лицензией CC BY-NC-ND license (http://creativecommons.org/licenses/by-nc-nd/4.0/). 


\section{Введение}

В данной работе мы будем рассматривать классы примитивно рекурсивных, общерекурсивных и частично рекурсивных функций, которые являются классами вычислимых функций, имеющими фундаментальное значение в математике $[1,2]$.

В 1947 году Рафаэль Робинсон в работе [3] доказал теорему о том, что все примитивно рекурсивные функции одной переменной, образующие класс $\mathfrak{F}_{\Pi p}^{(1)}$, и только они могут быть получены из двух функций

$$
s(x)=x+1, \quad q(x)=x-[\sqrt{x}]^{2}
$$

с помощью операций сложения + , суперпозиции * и итерации $i$, которые определяются следующим образом:

$$
\begin{gathered}
(f+g)(x)=f(x)+g(x), \\
(f * g)(x)=f(g(x)), \\
f=i(g), \text { где } f(0)=0 \text { и } f(n+1)=g(f(n)), n \geq 0 .
\end{gathered}
$$

Очевидно, итерация $i$ является частным случаем примитивной рекурсии [2]. Результатами этих операций также являются одноместные примитивно рекурсивные функции из $\mathfrak{F}_{\Pi р}^{(1)}$, т.е. класс $\mathfrak{F}_{\text {пр }}^{(1)}$ замкнут относительно указанных операций.

В 1950 году Джулия Робинсон в работе [4] доказала аналогичную теорему о том, что все общерекурсивные функции одной переменной, образующие класс $\mathfrak{F}_{\mathrm{op}}^{(1)}$, можно получить из тех же двух функций (1) с помощью операций сложения + , суперпозиции * и операции обращения ${ }^{-1}$, которая определяется следующим образом [2]:

$$
f^{-1}(x)=\mu_{y}(f(y)=x)
$$

где выражение $\mu_{y}(f(y)=x)$ определено и обозначает наименьшее значение $y$ такое, что $f(y)=x$, лишь в том случае, если это значение $y$ существует и при этом все значения $f(0), \ldots, f(y-1)$ определены и не равны $x$. Очевидно, если требуется оставаться в классе $\mathfrak{F}_{\text {op }}^{(1)}$, то операцию обращения $^{-1}$ следует считать частичной и применимой не ко всем функциям из $\mathfrak{F}_{\text {op }}^{(1)}$, а только лишь к тем, которые в результате дают снова всюду определённую, то есть общерекурсивную функцию. Это частный случай так называемой слабой минимизации [2, стр. 49].

Результатами так определённых операций являются одноместные общерекурсивные функции из $\mathfrak{F}_{\text {op }}^{(1)}$, т.е. класс $\mathfrak{F}_{\text {op }}^{(1)}$ замкнут относительно указанных операций.

Если же разрешить применять операцию ${ }^{-1}$ и в том случае, когда в результате может получаться частичная функция, то мы получим класс $\mathfrak{F}_{\text {чр }}^{(1)}$ всех одноместных частично рекурсивных функций. Этот класс $\mathfrak{F}_{\text {чp }}^{(1)}$, как следует из результата Дж. Робинсон [4], также порождается парой функций (1) с помощью операций,+ * $^{-1}$, где операция ${ }^{-1}$ применима уже ко всем функциям из $\mathfrak{F}_{\text {чр }}^{(1)}$.

В соответствии с этими результатами А. И. Мальцевым в 1961 году в работе [1] были введены три алгебры:

1) алгебра одноместных примитивно рекурсивных функций (алгебра ПРФ Рабаэля Робинсона)

$$
\mathfrak{A}_{\Pi \mathrm{p}}=<\mathfrak{F}_{\Pi \mathrm{p}}^{(1)} ;+, *, i>\text {; }
$$

2) алгебра одноместных общерекурсивных функций (алгебра ОРФ Джулии Робинсон)

$$
\mathfrak{A}_{\mathrm{op}}=<\mathfrak{F}_{\mathrm{op}}^{(1)} ;+,,^{-1}>\text {; }
$$


3) алгебра одноместных частично рекурсивных функций (алгебра ЧРФ Джулии Робинсон)

$$
\mathfrak{A}_{\text {чp }}=<\mathfrak{F}_{\text {чp }}^{(1)} ;+, *,^{-1}>
$$

и поставлена задача изучения свойств этих алгебр, в том числе проблема существования (или отсутствия) конечных базисов тождеств в многообразиях алгебр, порождённых указанными алгебрами. Заметим, что, как следует из результатов Р. Робинсона и Дж. Робинсон $[3,4]$, каждая из алгебр $\mathfrak{A}_{\Pi р}$,

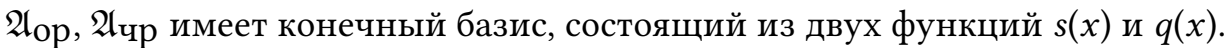

Цель данной статьи - показать, что ни в одном из многообразий $\mathfrak{M}_{п р}, \mathfrak{M}_{\mathrm{op}}, \mathfrak{M}_{\text {чр }}$, порождённых алгебрами $\mathfrak{A}_{\text {пр }}, \mathfrak{A}_{\text {ор }}, \mathfrak{A}_{\text {чр }}$ соответственно, конечного базиса тождеств не существует.

\section{1. Алгебра ПРФ Рафаэля Робинсона}

Пусть $\mathfrak{M}_{\text {пр }}$ - многообразие сигнатуры $\Omega_{\text {пр }}=<+, *, i>$, порождённое алгеброй $\mathfrak{A}_{\text {пр}}$, и пусть $\mathfrak{R}$

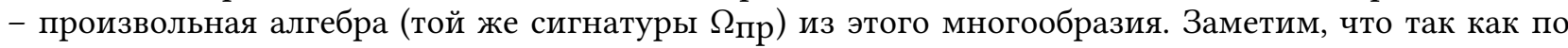
предположению алгебра $\mathfrak{R}$ принадлежит многообразию $\mathfrak{M}_{\text {пр }}$, в $\mathfrak{R}$ должны быть истинными все тождества многообразия $\mathfrak{M}_{\text {пр }}$.

Термы в алгебре $\mathfrak{R}$ определим следующим образом:

1) переменные $v$ и $v_{n}$ суть термы для любого натурального числа $n$;

2) пусть $t_{1}$ и $t_{2}-$ термы, тогда $\left(t_{1}+t_{2}\right),\left(t_{1} * t_{2}\right), i\left(t_{1}\right)$ - тоже термы.

Для уменьшения количества скобок в записи термов будем придерживаться следующей иерархии операций: высший приоритет у унарной операции итерации $i$, затем идёт операция суперпозиции * и, наконец, операция + с самым низким приоритетом. Учитывая, что операции сложения и суперпозиции ассоциативны, а операция сложения коммутативна, замечаем, что любой терм $t$ может быть представлен в виде

$$
t_{1}+t_{2}+\ldots+t_{n}
$$

где $n \geq 1$ и каждый терм $t_{j}$ - это либо суперпозиция двух каких-то термов, либо итерация некоторого терма, либо простая переменная, $j=1,2, \ldots, n$.

Если терм $t$ не является суммой других термов, то такой терм назовём одночленом. Одночлен вида $i(t)$ назовём $i$-замкнутым термом (для любого терма $t$ ). Очевидно, любой одночлен - это либо суперпозиция нескольких термов $t_{1} * \ldots * t_{k}, k \geq 2$, либо $i$-замкнутый терм $i(t)$, либо простая переменная $v$ (возможно, с индексом).

Терм $t=t_{1}+t_{2}+\ldots+t_{n}, n \geq 1$, где каждый терм $t_{j}, 1 \leq j \leq n$, является одночленом, будем называть многочленом, а число $n$ - его аддитивным рангом $\operatorname{ar}(t)$.

Пример: $\operatorname{ar}\left(v_{1}+v_{1} *\left(v_{2}+v_{3}+v_{4}\right)+i v_{1}\right)=3$.

Заметим, что если для некоторого терма $t \operatorname{ar}(t)=1$, то терм $t$ является одночленом.

Значение терма $t\left(v_{1}, \ldots, v_{n}\right), n \geq 1$, в некоторой алгебре $\mathfrak{R}$ из многообразия $\mathfrak{M}_{\text {пр }}$, получающееся при подстановке вместо переменных $v_{j}$ элементов $a_{j}$ из основного множества алгебры $\mathfrak{R}$, обозначим

$$
t\left(a_{1}, \ldots, a_{n}\right)_{\Re} .
$$

Тождество в алгебре $\mathfrak{R}$ многообразия $\mathfrak{M}_{\text {пр }}$ - это запись

$$
t_{1}\left(v_{1}, \ldots, v_{n}\right)=t_{2}\left(v_{1}, \ldots, v_{n}\right)
$$

где $t_{1}$ и $t_{2}$ - термы в алгебре $\Re$, каждый из которых, возможно, зависит фиктивно от некоторых переменных из списка $v_{1}, \ldots, v_{n}, n \geq 1$, при этом

$$
t_{1}\left(a_{1}, \ldots, a_{n}\right)_{\mathfrak{R}}=t_{2}\left(a_{1}, \ldots, a_{n}\right)_{\mathfrak{R}}
$$


при любых значениях $a_{j}$ (из носителя алгебры $\mathfrak{R}$ ) переменных $v_{j}, 1 \leq j \leq n$.

Примеры тождеств в алгебре $\mathfrak{R}$ :

$$
\begin{gathered}
v_{1}+v_{2}=v_{2}+v_{1} \\
i\left(i v_{1}+i v_{2}\right)=i\left(i v_{3}\right) .
\end{gathered}
$$

Аддитивный ранг тождества $t_{1}=t_{2}$ в алгебре $\mathfrak{R}$ многообразия $\mathfrak{M}_{\text {пр }}$ - это наибольший из аддитивных рангов термов $t_{1}$ и $t_{2}$.

Теорема 1. В многообразии алгебр $\mathfrak{M}_{n p}$, порождённом алгеброй примитивно рекурсивных функций Рафаэля Робинсона $\mathfrak{A}_{n p}=<\mathfrak{F}_{n p}^{(1)} ;+$, ,,$i>$, конечного базиса тождеств не существует.

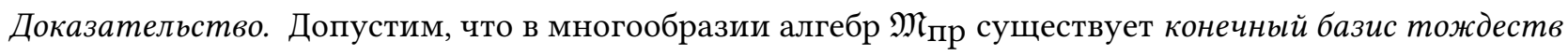
$\Sigma_{п р}$, т.е. конечный набор тождеств в сигнатуре $\Omega_{\Pi р}$, из которых выводимы все тождества, истинные в любой алгебре многообразия алгебр $\mathfrak{M}_{п р}$.

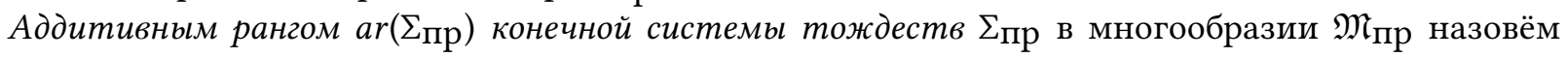
максимальный аддитивный ранг тождеств, входящих в $\Sigma_{п р}$.

Так как в алгебре $\mathfrak{A}_{п р}$ справедливы тождества, выражающие коммутативность и ассоциативность операции сложения, это позволяет опускать лишние скобки в записи термов и игнорировать порядок слагаемых в суммах одночленов в записи термов в любой алгебре многообразия $\mathfrak{M}_{п р}$.

Далее рассмотрим ещё одну алгебру

$$
\mathfrak{B}_{\Pi p}=<\mathfrak{F}_{\Pi p}^{(1)} ;+, *, i>
$$

у которой носитель $\mathfrak{F}_{\Pi p}^{(1)}$ - то же самое множество всех одноместных примитивно рекурсивных функций, как и у алгебры $\mathfrak{A}_{\Pi р}$, такая же сигнатура $\Omega_{п р}$, а определение термов совпадает с определением термов в алгебре $\mathfrak{R}$.

Все соглашения относительно старшинства операций в алгебре $\Re$ остаются в силе и для алгебры $\mathfrak{B}_{\text {пр, }}$ равно как и замечание о строении термов. Таким образом, можно утверждать, что любой

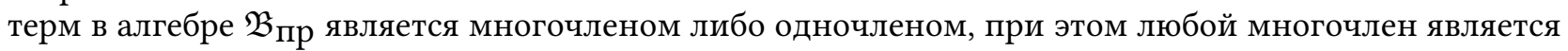
суммой двух или более одночленов.

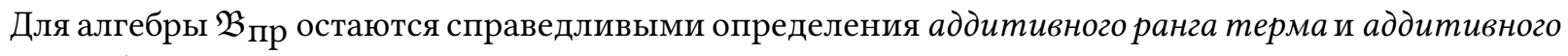
ранга тождества, так как они не зависят от семантики операций.

Что касается определений операций + и * в алгебре $\mathfrak{B}_{n р}$, то они точно такие же, как и для

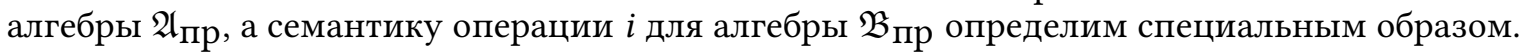

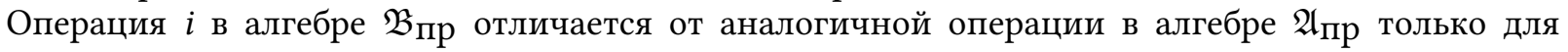
значений термов с «достаточно большими» аддитивными рангами.

Значения термов в алгебре $\mathfrak{A}_{n p}$.

1. Если терм $t$ - простая предметная переменная $v$ или $v_{n}$, то значением терма $t$ в алгебре $\mathfrak{A}_{\Pi р}$ будет $f$ для любой функции $f$ из $\mathfrak{F}_{\text {пр }}^{(1)}$.

2. Пусть $t_{1}\left(v_{1}, \ldots, v_{n}\right)$ и $t_{2}\left(u_{1}, \ldots, u_{m}\right)$ - термы. Тогда значениями термов

$$
t_{1}\left(v_{1}, \ldots, v_{n}\right)+t_{2}\left(u_{1}, \ldots, u_{m}\right)
$$

и

$$
t_{1}\left(v_{1}, \ldots, v_{n}\right) * t_{2}\left(u_{1}, \ldots, u_{m}\right)
$$

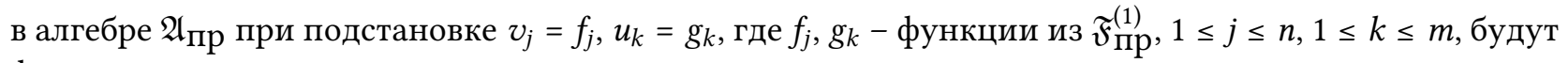
функции

$$
t_{1}\left(f_{1}, \ldots, f_{n}\right)+t_{2}\left(g_{1}, \ldots, g_{m}\right)
$$


и

$$
t_{1}\left(f_{1}, \ldots, f_{n}\right) * t_{2}\left(g_{1}, \ldots, g_{m}\right)
$$

соответственно.

3. Определим значение терма $i(t)$ для любого терма $t$ в алгебре $\mathfrak{A}_{п р}$. Как было показано ранее,

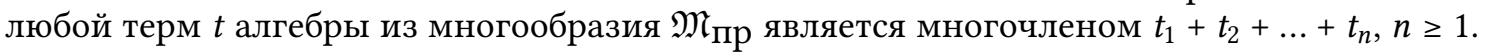

Предположим, что терм $t$ зависит от переменных $v_{1}, \ldots, v_{k}, k \geq 1$, и пусть функции $f_{1}, \ldots, f_{k}$ из $\mathfrak{F}_{\Pi р}^{(1)}$ являются значениями этих переменных. Можно считать, что каждый терм $t_{j}$ зависит от всех переменных $v_{1}, \ldots, v_{k}$ (возможно, что от некоторых из них $t_{j}$ зависит фиктивно). Тогда значением терма $t\left(v_{1}, \ldots, v_{k}\right)=i\left(t_{1}\left(v_{1}, \ldots, v_{k}\right)+\ldots+t_{n}\left(v_{1}, \ldots, v_{k}\right)\right)$ будет

$$
t\left(f_{1}, \ldots, f_{k}\right)_{\mathfrak{A}_{\Pi p}}=i\left(t_{1}\left(f_{1}, \ldots, f_{k}\right)+\ldots+t_{n}\left(f_{1}, \ldots, f_{k}\right)\right) .
$$

Значения термов в алгебре $\mathfrak{B} n p$.

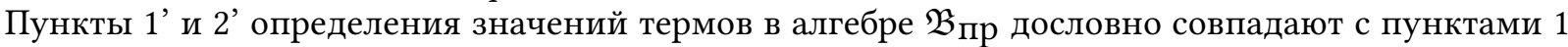
и 2 определения значений термов в алгебре $\mathfrak{A}_{п р}$.

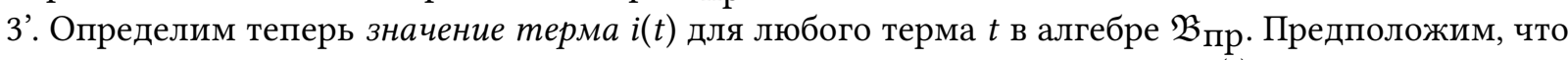
терм $t$ зависит от переменных $v_{1}, \ldots, v_{k}, k \geq 1$, и пусть функции $f_{1}, \ldots, f_{k}$ из $\mathfrak{F}_{\text {пр }}^{(1)}$ являются любыми значениями этих переменных. Терм $t$, как и в алгебре $\mathfrak{A}_{п р}$, будет суммой $n$ одночленов:

$$
t\left(v_{1}, \ldots, v_{k}\right)=t_{1}\left(v_{1}, \ldots, v_{k}\right)+\ldots+t_{n}\left(v_{1}, \ldots, v_{k}\right), n \geq 1 .
$$

Тогда значением терма $i\left(t\left(v_{1}, \ldots, v_{k}\right)\right)_{\mathfrak{B}_{\Pi р}}$ в алгебре $\mathfrak{B}_{\Pi р}$ будет

$$
\begin{array}{r}
i\left(t\left(f_{1}, \ldots, f_{k}\right)\right)_{\mathfrak{A}_{\Pi р}}, \text { если } n \leq \operatorname{ar}\left(\Sigma_{\Pi р}\right), \\
i\left(t\left(f_{1}, \ldots, f_{k}\right)+1\right)_{\mathfrak{A}_{\Pi р}}, \text { если } n>\operatorname{ar}\left(\Sigma_{\Pi р}\right) .
\end{array}
$$

Отметим следующие очевидные утверждения, вытекающие из введённых определений.

1. Тождество $t_{1}=t_{2}$, аддитивный ранг которого не превышает $\operatorname{ar}\left(\Sigma_{\Pi р}\right)$, истинно в алгебре $\mathfrak{A}_{\Pi р}$ тогда и только тогда, когда оно истинно в алгебре $\mathfrak{B}_{\text {пр. }}$.

2. Конечная система тождеств $\Sigma_{\Pi р}$, истинная в алгебре $\mathfrak{A}_{\Pi р}$, истинна и в алгебре $\mathfrak{B}_{\Pi р}$.

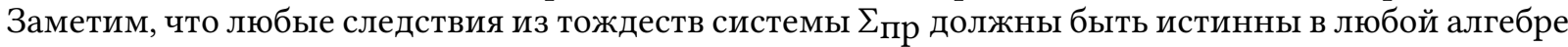
многообразия $\mathfrak{M}_{п р}$, включая алгебру $\mathfrak{B}_{\Pi р}$.

Очевидно, все термы $t_{1}$ и $t_{2}$, входящие в тождества $t_{1}=t_{2}$ системы $\Sigma_{\Pi р}$, имеют в алгебре $\mathfrak{B}_{\Pi р}$ такие же значения, как и в алгебре $\mathfrak{A}_{п р}$, то есть, как было сказано, алгебра $\mathfrak{B}_{п р}$ принадлежит многообразию $\mathfrak{M}_{п р}$. Это означает, что все тождества, выводимые из тождеств системы $\Sigma_{п р}$, должны быть истинными как в алгебре $\mathfrak{A}_{\Pi р}$, так и в алгебре $\mathfrak{B}_{\Pi р}$.

Рассмотрим серию тождеств в алгебре $\mathfrak{A}_{\Pi р}$ вида

$$
i\left(i v_{1}+\ldots+i v_{n}\right)=i\left(i v_{n+1}\right), n \geq 1 .
$$

Это так называемые «нулевые» тождества [5]. Нетрудно видеть, что при любых значениях переменных $v_{1}, \ldots, v_{n+1}$ и для любого натурального числа $n \geq 1$ значения термов в левой и в правой частях выражения (12) равны функции-константе 0.

Тождества данной серии истинны в алгебре $\mathfrak{A}_{п р}$, а значит, должны быть истинны в любой алгебре многообразия $\mathfrak{M}_{\Pi р}$.

Однако, равенство $i\left(i v_{1}+\ldots+i v_{n}\right)=i\left(i v_{n+1}\right)$ при $n>\operatorname{ar}\left(\Sigma_{\Pi р}\right)$, очевидно, является тождеством в

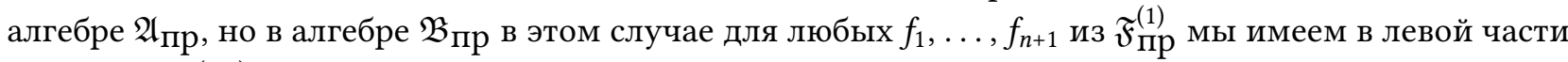
выражения (12) значение терма

$$
i\left(i f_{1}+\ldots+i f_{n}+1\right) \neq 0
$$


а в правой части выражения (12) мы имеем 0, так как для любой функции $f$ из $\mathfrak{F}_{\Pi р}^{(1)}$ значение i(if $)_{\mathfrak{B} \Pi p}=0$.

Таким образом, видим, что выражение

$$
i\left(i v_{1}+\ldots+i v_{n}\right)=i\left(i v_{n+1}\right)
$$

при $n>\operatorname{ar}\left(\Sigma_{\text {пр }}\right)$ не является тождеством в алгебре $\mathfrak{B}_{\text {пр. }}$

Полученное противоречие означает, что предположение о существовании конечного базиса

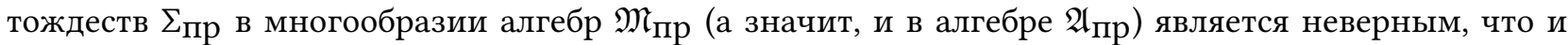
требовалось доказать.

Следствие 1. В алгебре Рафаэля Робинсона одноместных примитивно рекурсивных функций $\mathfrak{A}_{n p}=<\mathfrak{F}_{n p}^{(1)} ;+, *, i>$ конечного базиса тождеств не существует.

Следствие 2. В алгебре $\mathfrak{A}_{n p}^{+}=<\mathfrak{F}_{n p}^{(1)} ;+, i>$ конечного базиса тождеств не существует.

Обозначим через $\mathfrak{F}^{(1)}$ множество всех одноместных теоретико-числовых функций, определённых на множестве натуральных чисел и со значениями в том же множестве.

Следствие 3. В алгебрах одноместных теоретико-числовых функций $<\mathfrak{F}^{(1)} ;+, *, i>u<\mathfrak{F}^{(1)} ;+, i>$ конечного базиса тождеств не существует.

\section{2. Алгебра ЧРФ Джулии Робинсон}

Теперь перейдём к алгебре всех одноместных частично рекурсивных функций $\mathfrak{A}_{\text {чр }}=<\mathfrak{F}_{\text {чр }}^{(1)} ;+,{ }^{-1}>$. Операции сложения + и суперпозиции * частично рекурсивных функ-

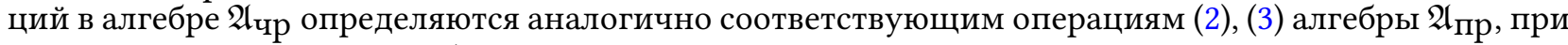
этом операция обращения ${ }^{-1}$ в алгебре $\mathfrak{A}_{\text {чр }}$, в отличие от алгебры $\mathfrak{A}_{\text {ор }}$, будет всюду определённой, так как результатом применения этой операции к любой частично рекурсивной функции будет некоторая частично рекурсивная функция из алгебры $\mathfrak{A}_{\text {чр. }}$

Схема доказательства для этого случая похожа на рассуждения в Теореме 1 , но имеет некоторые особенности.

Теорема 2. В многообразии алгебр Мчр, порождённом алгеброй частично рекурсивных функций Джулии Робинсон $\mathfrak{A}_{\text {чр }}=<\mathfrak{F}_{\text {чp }}^{(1)} ;+,{ }^{-1}>$, конечного базиса тождеств не существует.

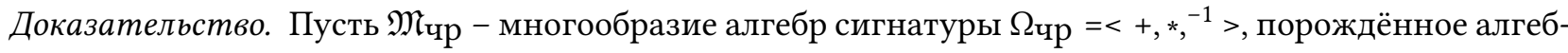
рой $\mathfrak{A}_{\text {чр }}$ и пусть $\mathfrak{G}$ - произвольная алгебра из этого многообразия.

Термы в алгебре $\mathfrak{G}$ определяются обычным образом:

1) переменные $v$ и $v_{n}$ суть термы для любого натурального числа $n$;

2) пусть $t_{1}$ и $t_{2}-$ термы, тогда $\left(t_{1}+t_{2}\right),\left(t_{1} * t_{2}\right),\left(t_{1}\right)^{-1}-$ тоже термы.

Как и в Теореме 1 , в записи термов будем придерживаться следующей иерархии операций: высший приоритет у одноместной операции обращения функции ${ }^{-1}$, затем идёт операция суперпозиции * и, наконец, операция + с самым низким приоритетом. Очевидно, любой терм $t$ в алгебре

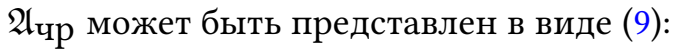

$$
t_{1}+t_{2}+\ldots+t_{n}
$$

где $n \geq 1$ и каждый терм $t_{j}$ - это либо суперпозиция двух каких-то термов, либо обращение некоторого терма, либо простая переменная, $j=1,2, \ldots, n$. 
Определим ранг терма $t$ по суперпозиции $s r(t)$ :

1) $\operatorname{sr}(v)=0$, где $v$ - простая переменная (возможно, с индексом);

2) пусть $t_{1}$ и $t_{2}$ - термы, тогда

$$
\begin{gathered}
s r\left(t_{1}+t_{2}\right)=s r\left(t_{1}\right)+s r\left(t_{2}\right), \\
s r\left(t_{1} * t_{2}\right)=s r\left(t_{1}\right)+s r\left(t_{2}\right)+1, \\
s r\left(\left(t_{1}\right)^{-1}\right)=s r\left(t_{1}\right) .
\end{gathered}
$$

Заметим, что если для некоторого терма $t s r(t)=n$, то в записи терма $t$ присутствуют ровно $n$ символов операции суперпозиции *, $n \geq 0$.

Пример: $\operatorname{sr}\left(v_{1}+\left(v_{1} * v_{2}\right) * v_{1}+\left(v_{2} * v_{1}\right)^{-1}\right)=3$.

Значение терма $t\left(v_{1}, \ldots, v_{n}\right), n \geq 1$, в некоторой алгебре $\mathfrak{G}$ из многообразия Мчр, получающееся при подстановке вместо переменных $v_{j}$ элементов $a_{j}$ из основного множества алгебры $\mathfrak{G}$, как и прежде, будем обозначать

$$
t\left(a_{1}, \ldots, a_{n}\right)_{\mathfrak{G}} .
$$

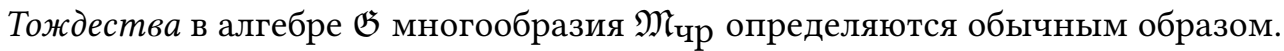

Примеры тождеств в алгебре $\mathfrak{G}$

$$
\begin{gathered}
\left(v_{1}+v_{2}\right)+v_{3}=v_{1}+\left(v_{2}+v_{3}\right) ; \\
\left(v_{1} * v_{2}\right) * v_{3}=v_{1} *\left(v_{2} * v_{3}\right) ; \\
v_{1}+v_{2}=v_{2}+v_{1} ; \\
v^{-1} * v * v^{-1}=v^{-1} .
\end{gathered}
$$

Ранг по суперпозищии тождества $t_{1}=t_{2}$ в многообразии $\mathfrak{M}_{\text {чр }}$ - это наибольший из рангов по суперпозиции $\operatorname{sr}\left(t_{1}\right)$ и $s r\left(t_{2}\right)$.

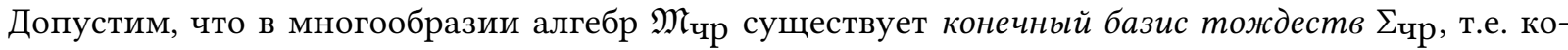

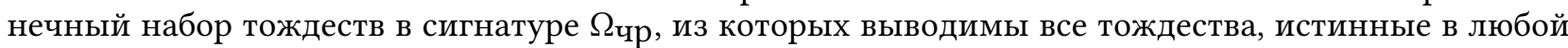
алгебре многообразия алгебр $\mathfrak{M}_{\text {чр }}$.

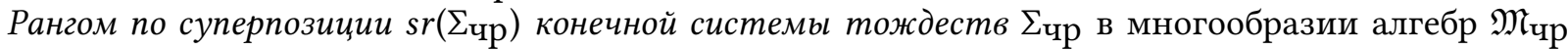
назовём максимальный ранг по суперпозиции тождеств, входящих в $\sum_{\text {чр. }}$

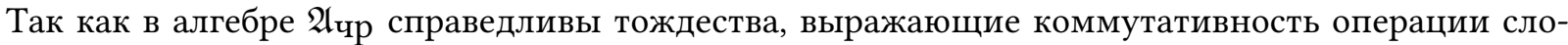
жения (15) и ассоциативность операций сложения (13) и суперпозиции (14), то это, как и ранее, позволяет опускать лишние скобки в записи термов в любой алгебре многообразия Мчр.

Рассмотрим ещё одну алгебру

$$
\mathfrak{B}_{\text {чp }}=<\mathfrak{F}_{\text {чp }}^{(1)} ;+, *,^{-1}>
$$

у которой носитель $\mathfrak{F}_{\text {чp }}^{(1)}$ - то же самое множество всех одноместных частично рекурсивных функций,

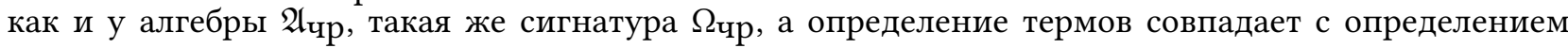
термов в алгебре $\mathfrak{G}$.

Все соглашения относительно старшинства операций в алгебре $\mathfrak{G}$ остаются в силе и для алгеб-

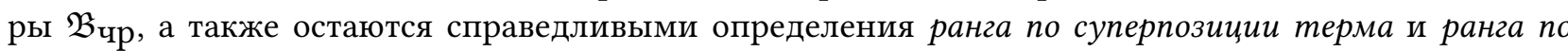
суперпозиции тождества, так как они не зависят от семантики операций.

Что касается определений операций + и * в алгебре $\mathfrak{B}_{\text {чp }}$, то они точно такие же, как и для алгебры

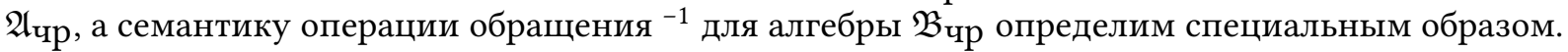

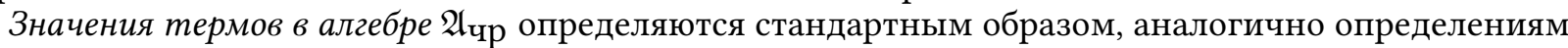

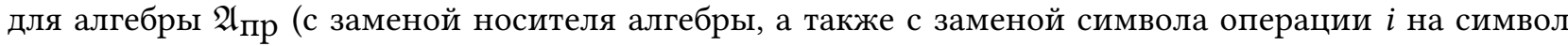
операции $^{-1}$ ). 
Значения термов в алгебре $\mathfrak{B}$ чр.

Определения значений термов для простой переменной $v$ (возможно, с индексом), а также для

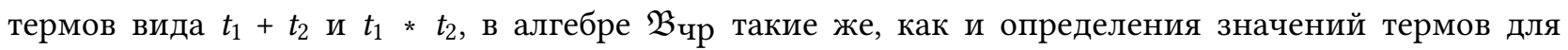
аналогичных операций в алгебре $\mathfrak{A}_{\text {чр. }}$.

Определим теперь значение терма $t^{-1}$ для любого терма $t$ в алгебре $\mathfrak{B}$ чр. Предположим, что терм $t$ зависит от переменных $v_{1}, \ldots, v_{k}, k \geq 1$, и пусть функции $f_{1}, \ldots, f_{k}$ из $\mathfrak{F}_{\text {чр }}^{(1)}$ являются любыми значениями этих переменных. Тогда значением терма $\left(t^{-1}\left(v_{1}, \ldots, v_{k}\right)\right)_{\mathfrak{B}}$ в алгебре $\mathfrak{B}$ чр будет

$$
\begin{array}{r}
\left(\left(t\left(f_{1}, \ldots, f_{k}\right)\right)^{-1}\right)_{\mathfrak{A}_{ч p}}, \text { если } \operatorname{sr}(t) \leq \operatorname{sr}\left(\sum_{\mathrm{up}}\right), \\
\left(\left(t\left(f_{1}, \ldots, f_{k}\right)+1\right)^{-1}\right)_{\mathfrak{A}_{\mathrm{up}}}, \text { если } \operatorname{sr}(t)>\operatorname{sr}\left(\sum_{\mathrm{up}}\right) .
\end{array}
$$

Отметим следующие очевидные утверждения, вытекающие из введённых определений.

1. Тождество $t_{1}=t_{2}$, ранг по суперпозиции которого не превышает $s r\left(\sum\right.$ чр), истинно в алгебре

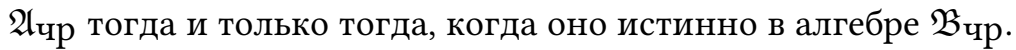

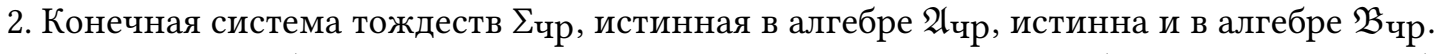

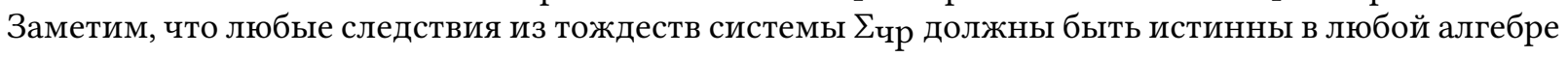
многообразия $\mathfrak{M}_{\text {чр }}$, включая алгебру $\mathfrak{B}_{\text {чр }}$.

Очевидно, все термы $t_{1}$ и $t_{2}$, входящие в тождества $t_{1}=t_{2}$ системы $\sum_{\text {чр}}$, имеют в алгебре $\mathfrak{B}_{\text {чр }}$

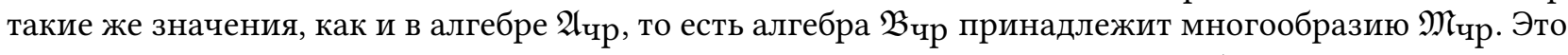
означает, что все тождества, выводимые из тождеств системы $\sum_{\text {чр}}$, должны быть истинными как в алгебре $\mathfrak{A}_{\text {чр }}$, так и в алгебре $\mathfrak{B}_{\text {чр }}$

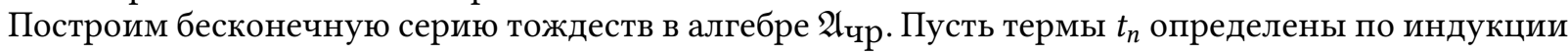
следующим образом:

$$
\begin{array}{r}
t_{0}(v)=v^{-1} \\
t_{n+1}(v)=t_{n}(v) * v * v^{-1}, n \geq 1
\end{array}
$$

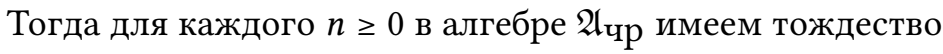

$$
\left(t_{n}(v) * v * v^{-1}\right)^{-1}=\left(v^{-1}\right)^{-1} .
$$

Нетрудно видеть, в частности,что при $n=0$ - это тождество

$$
\left(v^{-1} * v * v^{-1}\right)^{-1}=\left(v^{-1}\right)^{-1},
$$

получающееся из тождества (16), которое приведено в [2] на стр. 50, а

$$
\operatorname{sr}\left(t_{n}(v)\right)=2 n, n \geq 0 .
$$

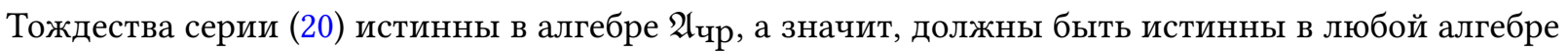
многообразия алгебр $\mathfrak{M}_{\text {чр }}$.

Однако, при $\operatorname{sr}\left(t_{n}(v)\right)>\operatorname{sr}\left(\sum_{\text {чр }}\right)$ равенство $\left(t_{n}(v) * v * v^{-1}\right)^{-1}=\left(v^{-1}\right)^{-1}$ является тождеством в

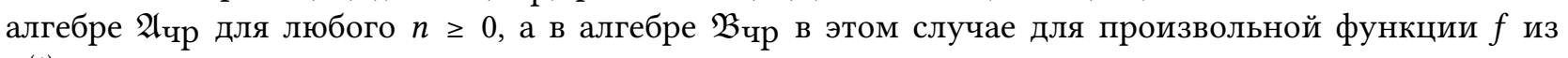
$\mathfrak{F}_{\text {чр }}^{(1)}$ мы имеем в левой части выражения (20) значение терма $\left(t_{n}(f) * f * f^{-1}+1\right)^{-1}$, а в правой части выражения (20) мы имеем $\left(f^{-1}\right)^{-1}$, т.е. равенство $(20)$ в этом случае нарушается в алгебре $\mathfrak{B}$ чр (например, для $f(x)=x$ ).

Таким образом, видим, что выражение

$$
\left(t_{n}(v) * v * v^{-1}\right)^{-1}=\left(v^{-1}\right)^{-1}
$$


при $2(n+1)>\operatorname{sr}\left(\sum_{\text {чp }}\right)$ не является тождеством в алгебре $\mathfrak{B}_{\text {чр. }}$

Полученное противоречие означает, что предположение о существовании конечного базиса

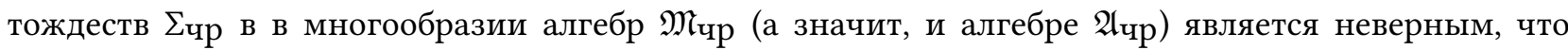
и требовалось доказать.

Следствие 4. В алгебре Джулии Робинсон одноместных частично рекурсивных функций $\mathfrak{A}_{\text {чр }}=<\mathfrak{F}_{\text {чp }}^{(1)} ;+,{ }^{-1}>$ конечного базиса тождеств не существует.

Следствие 5. В алгебре $\mathfrak{A}_{\text {чp }}^{*}=<\mathfrak{F}_{\text {чp }}^{(1)} ;{ }^{-1}>$ конечного базиса тождеств не существует.

Обозначим через $\mathfrak{F}_{ч}^{(1)}$ множество всех одноместных частичных теоретико-числовых функций, определённых на подмножествах множества натуральных чисел и со значениями в множестве натуральных чисел.

Следствие 6. В алгебрах одноместных частичных теоретико-числовых функций $<\mathfrak{F}_{u}^{(1)} ;+, *,{ }^{-1}>$ $u<\mathfrak{F}_{u}^{(1)} ;{ }^{-1},{ }^{-1}$ конечного базиса тождеств не существует.

\section{3. Алгебра ОРФ Джулии Робинсон}

В заключение рассмотрим алгебру всех одноместных общерекурсивных функций Джулии Робинсон $\mathfrak{A}_{\mathrm{op}}=<\mathfrak{F}_{\text {op }}^{(1)} ;+,{ }^{-1}>$. Особенность этого случая в том, что алгебра $\mathfrak{A}_{\text {ор }}$ является частичной $[1,6]$, так как операция обращения функции ${ }^{-1}$ применима лишь к таким общерекурсивным функциям, которые в результате применения этой операции дают всюду определённые функции, т.е. операция $^{-1}$ является частичной. Для частичной алгебры $\mathfrak{A}_{\text {ор }}$ значение терма $t\left(v_{1}, \ldots, v_{n}\right), n \geq 1$, может быть неопределённым для некоторого набора значений переменных $a_{1}, \ldots, a_{n}$ из основно-

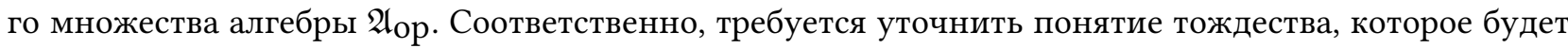
использоваться в этом случае.

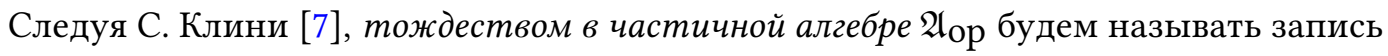

$$
t_{1}\left(v_{1}, \ldots, v_{n}\right) \approx t_{2}\left(v_{1}, \ldots, v_{n}\right)
$$

где $t_{1}$ и $t_{2}$ - термы в алгебре $\mathfrak{A}_{\mathrm{op}}$, каждый из которых, возможно, зависит фиктивно от некоторых переменных из списка $v_{1}, \ldots, v_{n}, n \geq 1$, такие, что при любых значениях $a_{j}$ (из носителя алгебры

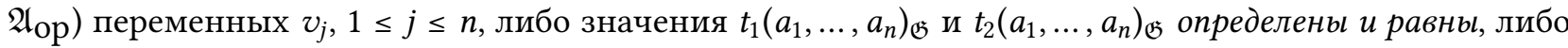
оба значения термов $t_{1}\left(a_{1}, \ldots, a_{n}\right)_{\mathfrak{G}}$ и $t_{2}\left(a_{1}, \ldots, a_{n}\right)_{\mathfrak{G}}$ не определены.

Примеры тождеств в частичной алгебре $\mathfrak{A}_{\mathrm{op}}$ :

$$
\begin{gathered}
\left(v_{1} * v_{2}\right) * v_{3} \approx v_{1} *\left(v_{2} * v_{3}\right), \\
v^{-1} * v * v^{-1} \approx v^{-1} .
\end{gathered}
$$

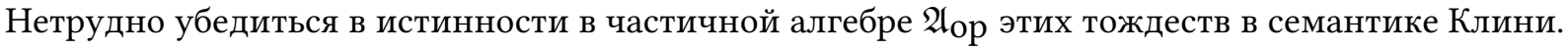

Пусть $\mathfrak{G}$ - произвольная частичная алгебра из многообразия $\mathfrak{M}$ ор, порождённого частичной алгеброй $\mathfrak{A}_{\text {op }}=<\mathfrak{F}_{\text {op }}^{(1)} ;+,{ }^{-1},{ }^{-1}$.

Термы в алгебре $\mathfrak{G}$ определяются так же, как и в алгебре $\mathfrak{A}_{\text {чр }}$, при этом любой терм $t$ в алгебре $\mathfrak{G}$ может быть представлен, как и в алгебре $\mathfrak{A}_{\text {чр }}$, в виде

$$
t_{1}+t_{2}+\ldots+t_{n}
$$

где $n \geq 1$ и каждый терм $t_{j}$ - это либо суперпозиция двух каких-то термов, либо обращение ${ }^{-1}$ некоторого терма, либо простая переменная, $j=1,2, \ldots, n$. 
Определение ранга терма $t$ по суперпозиции $\operatorname{sr}(t)$ в алгебре $\mathfrak{G}$ совпадает с соответствующим определением в алгебре $\mathfrak{A}_{\text {чр }}$, так же как и определение ранга по суперпозиции тождества $t_{1} \approx t_{2}$ в многообразии $\mathfrak{M}_{\mathrm{op}}$.

Как и в случае многообразия $\mathfrak{M}_{\text {чр }}$ рангом по суперпозиции $\operatorname{sr}\left(\Sigma_{\mathrm{op}}\right)$ конечной системы тождеств

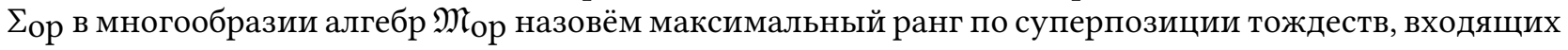
в $\Sigma_{\text {op. }}$

Будем опускать лишние скобки в записи термов в любой алгебре многообразия $\mathfrak{M}_{\mathrm{op}}$, пользуясь ассоциативностью операций + и * и коммутативностью операции + .

Дадим определение значения терма $t^{-1}$ для любого терма $t$ в частичной алгебре $\mathfrak{A}_{\mathrm{op}}$.

Предположим, что терм $t$ зависит от переменных $v_{1}, \ldots, v_{k}, k \geq 1$, и пусть функции $f_{1}, \ldots, f_{k}$ из $\mathfrak{F}_{\text {ор }}^{(1)}$ являются произвольными значениями этих переменных.

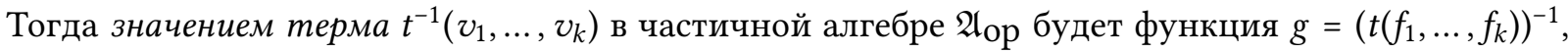
если она всюду определена на множестве натуральных чисел (т.е. является общерекурсивной функ-

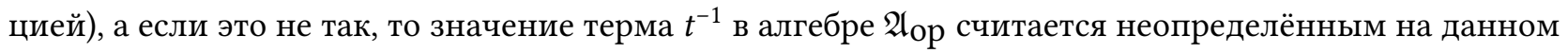
наборе $f_{1}, \ldots, f_{k}$ значений переменных $v_{1}, \ldots, v_{k}$.

Теорема 3. В многообразии частичных алгебр $\mathfrak{M}_{о р}$, порождённом частичной алгеброй одноместных общерекурсивных функиий Джулии Робинсон $\mathfrak{A}_{o p}=<\mathfrak{F}_{o p}^{(1)} ;+,{ }^{-1}>$, , конечного базиса тождеств (в определённом выше смысле) не существует.

Доказательство. Предположим, что в многообразии частичных алгебр $\mathfrak{M}_{\text {ор }}$ существует конечный базис тождеств $\Sigma_{\mathrm{op}}$, т.е. конечный набор тождеств в сигнатуре $\Omega_{\mathrm{op}}$, из которых выводимы все тождества, истинные в любой алгебре многообразия $\mathfrak{M}_{\mathrm{op}}$.

Рассмотрим частичную алгебру

$$
\mathfrak{B}_{\mathrm{op}}=<\mathfrak{F}_{\mathrm{op}}^{(1)} ;+,,^{-1}>
$$

у которой носитель $\mathfrak{F}_{\mathrm{Op}}^{(1)}$ - множество всех одноместных общерекурсивных функций, как и у алгебры $\mathfrak{A}_{\text {op }}$, такая же сигнатура $\Omega_{\mathrm{op}}$, а определение термов совпадает с определением термов в алгебре $\mathfrak{G}$.

Все соглашения относительно старшинства операций в алгебре $\mathfrak{G}$ остаются в силе и для алгебры $\mathfrak{B}_{\text {ор }}$, а также остаются справедливыми определения ранга терма по суперпозищии и ранга тождества по суперпозиции. Что касается определений операций + и * в частичных алгебрах $\mathfrak{A}_{\text {op }}$ и $\mathfrak{B}_{\text {op }}$, то они

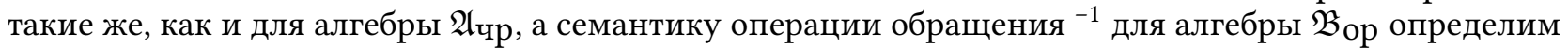
специальным образом.

Пусть терм $t$ зависит от переменных $v_{1}, \ldots, v_{k}, k \geq 1$, и пусть функции $f_{1}, \ldots, f_{k}$ из $\mathfrak{F}_{\text {ор }}^{(1)}$ являются значениями этих переменных.

Тогда значением терма $\left(t^{-1}\left(v_{1}, \ldots, v_{k}\right)\right)_{\mathfrak{B} \text { ор }}$ для данных значений $f_{1}, \ldots, f_{k}$ переменных $v_{1}, \ldots, v_{k}$

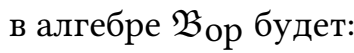

$$
\begin{array}{r}
\left(\left(t\left(f_{1}, \ldots, f_{k}\right)\right)^{-1}\right)_{\mathfrak{A}_{\mathrm{op}}}, \text { если } s r(t) \leq \operatorname{sr}\left(\sum_{\mathrm{op}}\right), \\
\left(\left(t\left(f_{1}, \ldots, f_{k}\right)+1\right)^{-1}\right)_{\mathfrak{A}_{\mathrm{op}}}, \text { если } \operatorname{sr}(t)>\operatorname{sr}\left(\Sigma_{\mathrm{op}}\right) .
\end{array}
$$

Отметим следующие очевидные утверждения, вытекающие из введённых определений.

1 . Тождество $t_{1} \approx t_{2}$, ранг по суперпозиции которого не превышает $\operatorname{sr}\left(\Sigma_{\mathrm{op}}\right)$, истинно в частичной

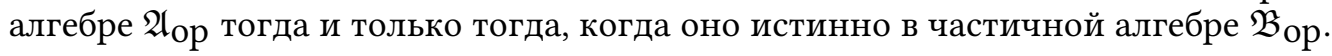

2. Конечная система тождеств $\Sigma_{\mathrm{op}}$, истинная в частичной алгебре $\mathfrak{A}_{\mathrm{op}}$, истинна и в частичной алгебре $\mathfrak{B}_{\text {op }}$.

Очевидно, все термы $t_{1}$ и $t_{2}$, входящие в тождества $t_{1} \approx t_{2}$ системы $\Sigma_{\text {ор }}$, имеют в алгебре $\mathfrak{B}_{\text {ор такие }}$ же значения, как и в алгебре $\mathfrak{A}_{\text {op }}$, то есть алгебра $\mathfrak{B}_{\text {op }}$, очевидно, принадлежит многообразию $\mathfrak{M}_{\text {op }}$. 
Это означает, что все тождества, выводимые из тождеств системы $\Sigma_{\mathrm{op}}$, должны быть истинными как в алгебре $\mathfrak{A}_{\text {op }}$, так и в алгебре $\mathfrak{B}_{\text {op }}$.

Рассмотрим серию равенств $t_{n}(v) * v * v^{-1} \approx v^{-1}, n \geq 0$, в произвольной частичной алгебре $\mathfrak{G}$ многообразия $\mathfrak{M}_{\mathrm{op}}$, где термы $t_{n}(v)$ определены по формулам (19), и $s r\left(t_{n}(v)\right)=2 n, n \geq 0$.

Нетрудно видеть, что эти равенства, а также их следствия

$$
\left(t_{n}(v) * v * v^{-1}\right)^{-1} \approx\left(v^{-1}\right)^{-1}, n \geq 0
$$

являются тождествами в семантике Клини в частичной алгебре $\mathfrak{A}_{\text {ор }}$ и, следовательно, должны быть истинны в любой алгебре многообразия $\mathfrak{M}$ ор.

Но в алгебре $\mathfrak{B}_{\mathrm{op}}$, в соответствии с определением (25), при $\operatorname{sr}\left(t_{n}(v)\right)>\operatorname{sr}\left(\Sigma_{\text {ор }}\right)$ равенство (26) нарушается, например, для функции $f(x)=x$ из $\mathfrak{F}_{\mathrm{op}}^{(1)}$. Таким образом, видим, что выражения $\left(t_{n}(v) * v * v^{-1}\right)^{-1} \approx\left(v^{-1}\right)^{-1}$ при $2(n+1)>\operatorname{sr}\left(\Sigma_{\mathrm{op}}\right)$ не являются тождествами в частичной алгебре $\mathfrak{B}_{\text {op. }}$.

Полученное противоречие означает, что предположение о существовании конечного базиса

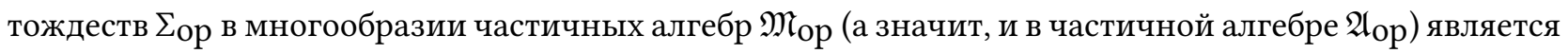
неверным, что и требовалось доказать.

Следствие 7. В частичной алгебре Джулии Робинсон одноместных общерекурсивных функций $\mathfrak{A}_{\text {ор }}=<\mathfrak{F}_{\text {op }}^{(1)} ;+,,^{-1}>$ конечного базиса тождеств не существует.

Следствие 8. В частичной алгебре $\mathfrak{A}_{o p}^{*}=<\mathfrak{F}_{o p}^{(1)} ;{ }^{-1}{ }^{-1}>$ конечного базиса тождеств не существует.

Следствие 9. В алгебрах одноместных теоретико-числовых функций $<\mathfrak{F}_{o p}^{(1)} ;+, *,{ }^{-1}>u<\mathfrak{F}_{\text {op }}^{(1)} ;{ }^{-1}>$ конечного базиса тождеств не существует.

\section{Заключение}

В работе решена проблема А. И. Мальцева существования конечного базиса тождеств в алгебрах одноместных рекурсивных функций. Показано, что в алгебрах примитивно рекурсивных и частично рекурсивных функций конечного базиса тождеств не существует. Так как алгебра одноместных общерекурсивных функций является частичной, для неё аналогичная проблема была решена в семантике Клини для тождеств.

\section{References}

[1] A. I. Mal'tsev, “Constructive algebras I”, Russian Mathematical Surveys, vol. 16, no. 3, pp. 77-129, 1961.

[2] A. I. Mal'tsev, Algoritmy i rekursivnye funktsii. Moscow: Nauka, 1965, In Russian.

[3] J. Robinson, "Primitive recursive functions", Proceedings of the American Mathematical Society, vol. 1, no. 6, pp. 703-718, 1950.

[4] R. M. Robinson, "Primitive recursive functions", Bulletin of the American Mathematical Society, vol. 53, no. 10, pp. 925-942, 1947.

[5] V. A. Sokolov, "Ob odnom klasse tozhdestv v algebre Robinsona", in 14-ya Vsesoyuznaya algebraicheskaya konferentsiya: tezisy dokladov, In Russian, vol. 2, Novosibirsk, 1977, pp. 123-124.

[6] P. M. Cohn, Universal Algebra. New York, Evanston, and London: Harper \& Row, 1965.

[7] A. Robinson, "Equational logic for partial functions under Kleene equality: a complete and an incomplete set of rules”, The fournal of Symbolic Logic, vol. 54, no. 2, pp. 354-362, 1989. 